\title{
Correlation of promoter hypermethylation in hTERT, DAPK and MGMT genes with cervical oncogenesis progression
}

\author{
DIMITRIOS ILIOPOULOS ${ }^{1,2^{*}}$, PAGONA OIKONOMOU $^{1,3^{*}}$, IOANNIS MESSINIS $^{4}$ and ASPASIA TSEZOU ASP $^{1,3,5}$ \\ ${ }^{1}$ Institute of Biomedical Research and Technology, Larissa, Greece; ${ }^{2}$ Department of Biological Chemistry \\ and Molecular Pharmacology, School of Medicine, Harvard University, Boston, MA, USA; \\ ${ }^{3}$ Laboratory of Cytogenetics and Medical Genetics, ${ }^{4}$ Departments of Obstetrics and Gynecology, \\ and ${ }^{5}$ Biology, University of Thessalia, Medical School, Larissa, Greece
}

Received January 22, 2009; Accepted March 30, 2009

DOI: $10.3892 /$ or_00000425

\begin{abstract}
DNA hypermethylation occurs during the multistep process of cervical carcinogenesis. We investigated whether the methylation status in the promoter region of a potential oncogene, the human telomerase reverse transcriptase (hTERT), and the tumor suppressor genes deathassociated protein kinase (DAPK) and $\mathrm{O}^{6}$-methylguanine DNA methyltransferase (MGMT), were able to distinguish the early from late stages of cervical oncogenesis. The methylation status in the promoter of these genes was analyzed using real-time MethyLight analysis in 115 cervical specimens, including normal, premalignant [atypical squamous epithelial cells (ASCUS), low-grade squamous intraepithelial lesions (LGSIL), high-grade squamous intraepithelial lesions (HGSIL)] and cancer specimens. Clinicopathological parameters (cytology, histology, grade, stage) were compared to the levels of pro-moter hypermethylation. We found that hTERT, MGMT and DAPK hypermethylation levels were increased during cervical oncogenesis progression. hTERT promoter hypermethylation was able to distinguish normal from cancer ( $\mathrm{p}=0.008)$, normal from premalignant $(\mathrm{p}=0.036)$, as well as premalignant from cervical cancer cases $(p=0.003)$. A significant association was also observed between all three genes and the grade of cervical cancer, with hTERT showing a better association $(\mathrm{p}<0.0001)$. Our data suggest that the combination of hTERT, MGMT, DAPK promoter hypermethylation could have a potential function as molecular biomarker of cervical oncogenesis progression.
\end{abstract}

Correspondence to: Professor Aspasia Tsezou, Department of Biology, Medical School, University of Thessalia, 22 Papakyriazi Street, 41222 Larissa, Greece

E-mail: atsezou@med.uth.gr

*Contributed equally

Key words: DNA methylation, telomerase, methylguanine DNA methyltransferase, death-associated protein kinase, human telomerase reverse transcriptase

\section{Introduction}

Cervical cancer $(\mathrm{CC})$ is a leading cause of cancer-related mortality in women worldwide $(1,2)$. Cervical carcinomas develop as a result of multiple genetic and epigenetic alterations that accumulate during time, with atypical squamous epithelial cells (ASCUS) in the uterine cervix progressing into low-grade (LGSIL) or high-grade (HGSIL) squamous intraepithelial lesions, which could lead to cancer development $(3,4)$. Converging evidence from epidemiological and molecular studies indicates that human papillomavirus (HPV) is the major causative agent in the development of cervical cancer (5-7). However, other factors might also be involved in cervical carcinogenesis, as the majority of patients infected with human papillomavirus will not develop cervical cancer.

Cytomorphological examination of cervical smears is most widely applied, although Pap smear has false negative rates of $2-40 \%$, due to a combination of sampling error, processing artifacts and the nature of subjective interpretation (8). HPV testing has been suggested to improve cervical cancer screening, however its specificity remains relatively low (9).

Detection of changes in DNA methylation, also termed epigenetic alterations, may offer an additional approach to cervical screening. It has been increasingly recognized that the $\mathrm{CpG}$ islands of a large number of genes that are unmethylated in normal tissues, are methylated to various degrees in multiple types of human cancer and particularly in gynecological cancer (10-15). In addition to the functional implications of gene inactivation in tumor development, these aberrant methylation patterns represent excellent targets for novel diagnostic approaches based on methylation-sensitive PCR techniques.

Epigenetic hypermethylation in the CpG- rich sequences in the promoter regions of a number of genes, including the potential oncogene hTERT and the suppressor genes DAPK and MGMT, has been recognized as an important change that takes place in cervical cancer (16). hTERT gene encodes the catalytic subunit of telomerase, an enzyme which plays a critical role in chromosome structure and function as well as in cancer development (17). Recently Cohen et al, determined that human telomerase consists of two molecules 
Table I. Histologic outcome and presence of HPV.

\begin{tabular}{|c|c|c|c|c|c|c|c|c|}
\hline \multirow{3}{*}{ Cytologic outcome } & \multirow{3}{*}{ No. of samples } & \multicolumn{3}{|c|}{ HPV } & \multicolumn{4}{|c|}{ Histological outcome } \\
\hline & & \multirow[t]{2}{*}{ Negative } & \multicolumn{2}{|c|}{ Positive } & \multirow[t]{2}{*}{$\mathrm{Neg}$} & \multirow[t]{2}{*}{ CIN1 } & \multirow[t]{2}{*}{ CIN2 } & \multirow[t]{2}{*}{$\mathrm{CIN} 3$} \\
\hline & & & Non-oncogenic & $\begin{array}{c}\text { Oncogenic } \\
(\mathrm{HPV}-16,18)\end{array}$ & & & & \\
\hline NormalL & 15 & 12 & 3 & - & - & - & - & - \\
\hline ASCUS & 12 & 7 & 4 & 1 & 9 & 3 & - & - \\
\hline LGSIL & 15 & 4 & 5 & 4 & - & 3 & 12 & - \\
\hline HGSIL & 12 & 1 & 3 & 8 & - & - & 2 & 10 \\
\hline Cancer & 61 & 29 & 8 & 26 & - & - & - & - \\
\hline Total & 115 & 53 & 23 & 39 & 9 & 6 & 14 & 10 \\
\hline
\end{tabular}

of each telomerase reverse transcriptase, telomerase RNA and dyskerin (18). DAPK is a proapoptotic gene that potentially inhibits metastasis by increasing the occurrence of apoptosis, while MGMT is a DNA repair gene, which encodes a DNArepair protein that removes alkyl groups from the $\mathrm{O}^{6}$ position of guanine, an important site of DNA (19-22).

The aim of the present study was to determine the methylation status in the promoter region of hTERT DAPK and MGMT genes in normal, premalignant and malignant cervical samples, in an effort to investigate whether this epigenetic event is involved in the process of cervical carcinogenesis.

\section{Material and methods}

Patients. A total of 115 cervical samples were studied including 15 normal cases (mean age $36 \pm 4.4$; range $24-62$ years), 39 cases (mean age $38.5 \pm 4.8$; range 18-54 years) with abnormal cytologic findings (premalignant) including 12 cases of ASCUS (mean age 40.2 \pm 5.1 ; range 24-50 years), 15 cases of LGSIL (mean age 35.6 \pm 4.6 ; range 21-54 years) and 12 cases of HGSIL (mean age 36.8 +5.2 ; range 29-47 years) as well as 61 cases of cervical carcinomas (mean age 46.2 \pm 5.4 ; range 26-62 years). Among premalignant samples 6/39 (15.4\%) were CIN 1, 14/39 (35.9\%) were CIN2 and 10/39 (25.6\%) were CIN3 (Table I).

Scrapings from normal samples were collected from women volunteers after a normal cytology test result and a negative HPV DNA test. Scrapings from cervical samples of premalignant cases were obtained at the Colposcopy Unit of University Hospital of Larissa in Greece. Cervical cancer samples were provided from US Biomax Inc cancer tissue bank collection (US Biomax Inc., MD, USA). The case selection was random and controlled for HPV status and age. The control group matched the above demographics. All abnormal samples were collected at the time of colposcopic evaluation for the management of previous abnormal cytology test results, including repeated cytological diagnosis of ASCUS or previous diagnosis of LGSIL or HGSIL. Cervical specimens were obtained from each woman in collection vials containing STE buffer ( $\mathrm{pH} 7.5,0.05 \mathrm{M}$ Tris $\mathrm{HCl}, \mathrm{NaCl}$ $0.1 \mathrm{M}$, EDTA $1 \mathrm{mM}$ ) using an endocervical cytobrush prior to the colposcopic examination and cervical biopsy and immediately after collection of the routine cervical sample. Presence of HPV in premalignant samples is shown in Table I. Cytological cases were assessed according to the Bethesda System.

Cervical cancer cases included 34 with stage I (mean age $39.5 \pm 5.3$; range 26-56 years), 15 with stage II (mean age $47.6 \pm 5.6$; range $31-62$ years) and 12 with stage III (mean age 57.7 \pm 5.7 ; range $41-62$ years). The stages of each cancer were established according to the International Federation of Gynecology and Obstetrics (FIGO) criteria. The study was double blinded. The specimens were immediately processed in the laboratory for DNA extraction and subsequent for HPV typing. The histological types and grades of tumor were classified according to WHO criteria. Study records were reviewed according to the Institutional Review Board guidelines.

$H P V$ analysis. Human genomic DNA was extracted from the scraped cervical cells using a proteinase K/phenol-chloroform protocol. HPV detection and genotyping were performed as previously described (23).

Real-time MethyLight analysis. To assess promoter hypermethylation, DNA was extracted using a proteinase $\mathrm{K} /$ phenol-chloroform protocol and was treated with bisulfite as described previously (24). After sodium bisulfite conversion, the methylation analysis was performed by the fluorescencebased, real-time PCR assay, MethyLight. Briefly, one set of primers designed specifically for bisulfite-converted DNA, was used: a methylated set for the gene of interest and a reference set, $B$-actin $(A C T B)$ to normalize for input DNA. Specificity of the reactions for methylated DNA was confirmed separately using SssI (New England Biolabs) treated human genomic DNA (heavily methylated). The percentage of fully methylated molecules at a specific locus was calculated by dividing the $G E N E$ :ACTB ratio of a sample by the GENE:ACTB ratio of SssI-treated human genomic DNA and multiplying by 100 . The abbreviation PMR (percentage of fully methylated reference) indicates this measurement. A gene was deemed methylated if the PMR value was $>0$. To verify the reproducibility of each assay, the normalized value 
Table II. Sequences of the primers used in MethyLight reactions.

\begin{tabular}{lllll}
\hline Gene & \multicolumn{1}{c}{ hTERT } & \multicolumn{1}{c}{ DAPK } & \multicolumn{1}{c}{ MGMT } & B-actin \\
\hline Forward & 5'-GCGTCGGAGG & 5'-TCGTCGTCGTT & 5'-CGAATATACTAA' & 5'-TGGTGATGGAGG \\
primers & TTAAGGTTGTT-3' & TCGGTTAGTT-3' & AACAACCCGCG-3 & AGGTTTAGTAAGT-3' \\
Reverse & 5'-CTCTCCAAAAT & 5'-TCCCTCCGAA & 5'-GTATTTTTTCGG & 5'-AACCAATAAAAC \\
primers & TACCGTACGCG-3' & ACGCTATCG-3' & GAGCGAGGC-3' & CTACTCCTCCCTTAA3' \\
Probes & 5'-FAM-AACTCGCTCG & 5'-FAM-CGACCATAAAC & 5'-FAM-AATCCTCGCGATA & 5'-6FAM-ACCACCACCCAACA \\
& CCCGCCGAA-BHQ-3' & GCCAACGCCG-TAMRA-3' & CGCACCGTTTACG-TAMRA-3' & CACAATAACAAACACA-TAMRA-3' \\
\hline
\end{tabular}

Table III. Frequency of DNA methylation of hTERT, MGMT and DAPK genes in cervical tissues.

\begin{tabular}{|c|c|c|c|c|c|c|c|}
\hline \multirow[b]{2}{*}{ Cases } & \multirow[b]{2}{*}{115} & \multicolumn{2}{|c|}{ hTERT } & \multicolumn{2}{|c|}{ MGMT } & \multicolumn{2}{|c|}{ DAPK } \\
\hline & & $\mathrm{M}(\%)$ & $\mathrm{U}(\%)$ & $\mathrm{M}(\%)$ & $\mathrm{U}(\%)$ & $\mathrm{M}(\%)$ & $\mathrm{U}(\%)$ \\
\hline Normal & 15 & $4(26.6)$ & $11(73.4)$ & $2(13.3)$ & 13 (86.7) & $0(0)$ & 15 (100) \\
\hline Precancerous & 39 & $18(46.2)$ & $21(53.8)$ & $11(39.2)$ & $28(60.8)$ & $22(56.4)$ & 13 (43.6) \\
\hline Cancer & 61 & $40(65.6)$ & $21(34.4)$ & $37(60.6)$ & $24(39.4)$ & $41(67.2)$ & $26(32.8)$ \\
\hline Stage I & 34 & $19(55.8)$ & $15(44.2)$ & $14(41.2)$ & $20(58.8)$ & $25(73.5)$ & $9(26.5)$ \\
\hline Stage II & 15 & $11(73.3)$ & $4(26.7)$ & $11(73.3)$ & $4(26.7)$ & $14(93.3)$ & $1(6.7)$ \\
\hline Stage III-IV & 12 & $10(83.3)$ & $2(16.7)$ & $12(100)$ & $0(0)$ & $12(100)$ & $0(0)$ \\
\hline
\end{tabular}

M, methylated; U, unmethylated.

(Gene:ACTB) of the standard sample was compared between the different PCR runs. The primers and probes that were used for the MethyLight reactions have been previously described (25-27) and are shown in Table II.

Statistical analysis. Data were analysed by $\chi^{2}$ test, unipaired t-test, Mann-Whitney U (MWU), Kruskal-Wallis, as well as analysis of variance (ANOVA) and the Fisher PLSD; the post-hoc test corrected for multiple comparisons, where applicable. Bonferroni corrections were also used to adjust for multiple comparisons among the three genes and clinicopathological parameters. A p-value $<0.05$ was considered as statistically significant. Statistical analysis was performed using the SPSS, version 12.0 for Windows (SPSS, Chicago, IL). Statistical analysis was performed using the SPSS 10 software. Diagnostic test characteristics were calculated by using the proportion of women with normal, premalignant and cancer cytological data.

\section{Results}

Frequency of promoter methylation in cervical specimens. To assess hTERT, MGMT, and DAPK hypermethylation status in normal, premalignant and cancerous cervical samples, we performed real-time QMSP (real-time quantitative methylation specific PCR) analysis. hTERT promoter methylation was detected in $26.6 \%$ of normal cases, $46.2 \%$ of premalignant $[41.7 \%(5 / 12)$ of ASCUS cases, $33.3 \%(5 / 15)$ of LGSILs, $66.7 \%$ (8/12) of HGSILs] and $65.6 \%$ of cancer cases (Table III).

Also, MGMT promoter methylation was found in $13.3 \%$ of normal cases, $28.2 \%$ of premalignant $[6.7 \%(1 / 12)$ of ASCUS cases, $6.7 \%(1 / 15)$ of LGSILs, $75 \%(9 / 12)$ of HGSILs] and $72.5 \%$ of cancer cases. DAPK promoter methylation was not detected in any of the normal cases, $66.7 \%$ of premalignant $[33.3 \%(4 / 12)$ of ASCUS cases, $66.7 \%(10 / 15)$ of LGSILs, $100 \%(12 / 12)$ of HGSILs] and $57.4 \%$ of cancer cases. Overall, these results suggest that there is an increase in the frequency of hTERT, MGMT and DAPK methylated samples during cervical oncogenesis progression.

Promoter hypermethylation in relation to cytological findings. Mean QMSP values were correlated with the cytological findings (Fig. 1). Mean normalized QMSP values of hTERT promoter methylation were 0.50 (range 0-4.3), 2.7 (range $0-13.2$ ) and 8.30 (range 0-41.2) in normal, premalignant and cancer cases, respectively. A significant difference was observed between normal and cancer $(\mathrm{p}=0.008)$ and between normal and premalignant $(\mathrm{p}=0.036)$ as well as between premalignant and cancer cases $(\mathrm{p}=0.003)$. Among premalignant cases mean normalized QMSP values of hTERT promoter methylation were 1.73 (range 0-6.7), 2.55 (range 0-11.2) and 4.02 (range 0-13.2) in ASCUS, LGSILs and HGSILs, respectively. A significant difference was observed between normal and cancer $(\mathrm{p}=0.008)$, normal and HGSILs $(\mathrm{p}=0.012)$, 


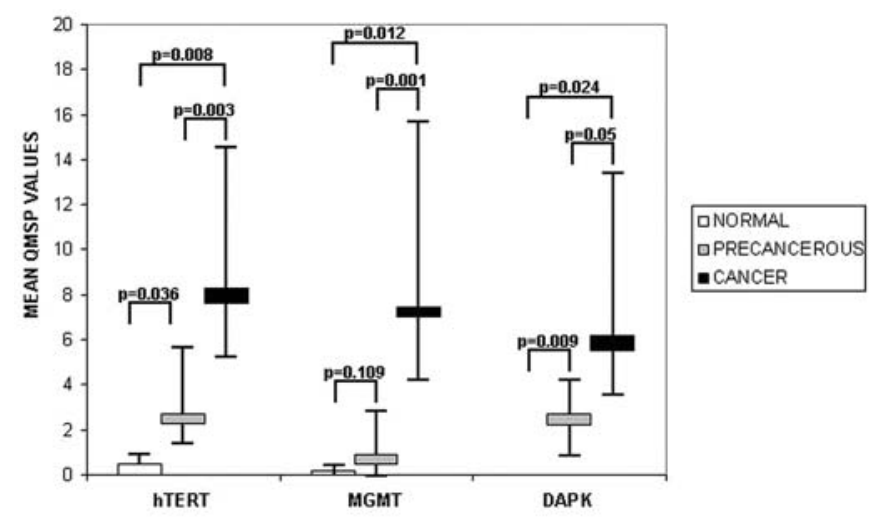

Figure 1. Mean QMSP values of hTERT, MGMT and DAPK promoter methylation levels in normal, premalignant and cancer sample and association with cervical oncogenesis progression. hTERT and DAPK promoter methylation is able to distinguish normal, premalignant and cervical cancer cases, while MGMT promoter methylation is able to distinguish premalignant and cancer cases but not between normal and premaligant cases.

ASCUS and cancer ( $\mathrm{p}=0.045)$, LGSILs and HGSILs $(\mathrm{p}=0.00)$, LGSILs and cancer $(\mathrm{p}=0.05)$ and between HGSIL and cancer specimens $(\mathrm{p}=0.04)$.

MGMT mean QMSP values were 0.16 (range 0-1.30), 1.18 (range 0-11.20) and 7.47 (range 0-45.4) in normal, premalignant and cancer cases, respectively. A significant difference was also observed between normal and cancer $(\mathrm{p}=0.012)$, as well as between premalignant and cancer cases $(\mathrm{p}=0.001)$. No significant differences were observed between normal and premalignant cases $(\mathrm{p}=0.109)$. Among premalignant samples MGMT mean QMSP values were 0.26 (range $0-3.1$ ), 0.28 (range 0-4.3) and 3.25 (range 0-11.2) in ASCUS, LGSIL and HGSIL cases, respectively. A significant difference was also observed between normal and cancer $(p=0.012)$, normal and HGSILs ( $\mathrm{p}=0.001)$, ASCUS and cancer $(\mathrm{p}=0.026)$, LGSILs and HGSILs $(\mathrm{p}=0.013)$, LGSILs and cancer $(\mathrm{p}=0.039)$, HGSIL and cancer $(\mathrm{p}=0.015)$ as well as between LGSILs and HGSILs $(\mathrm{p}=0.013)$ and ASCUS and HGSILs $(\mathrm{p}=0.006)$.

DAPK mean QSP values were 0.2.7 (range 0-21.2) and 5.4 (range 0-44.2) in normal, premalignant and cancer cases, respectively. A significant difference was also observed between normal and cancer $(\mathrm{p}=0.024)$, normal and premalignant cases $(\mathrm{p}=0.009)$ as well as premalignant and cancer $(\mathrm{p}=0.05)$. Among premalignant samples DAPK mean QSP values were 0.74 (range 0-3.1), 2.52 (range 0-7.7) and 5.1 (range 0-21.2) in ASCUS, LGSIL and HGSIL cases, respectively. A significant difference was observed between normal and cancer $(\mathrm{p}=0.024)$, normal and HGSILs ( $\mathrm{p}=0.002)$, ASCUS and cancer $(\mathrm{p}=0.008)$, ASCUS and HGSILs $(\mathrm{p}=0.019)$, as well as between normal and ASCUS $(\mathrm{p}=0.022)$, normal and LGSILs $(\mathrm{p}=0.001)$ and ASCUS and LGSILs $(\mathrm{p}=0.032)$. According to these results it seems that DAPK promoter hypermethylation is the best marker of cervical oncogenesis progression. The same correlations were found in all genes when ASCUS and LGSIL specimens were grouped together. When sensitivity values were evaluated, it was observed that the combination of hTERT, MGMT and DAPK a

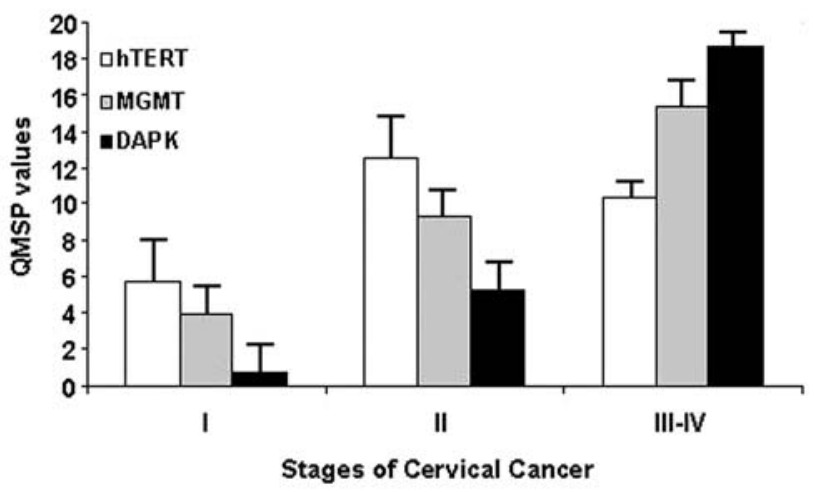

b

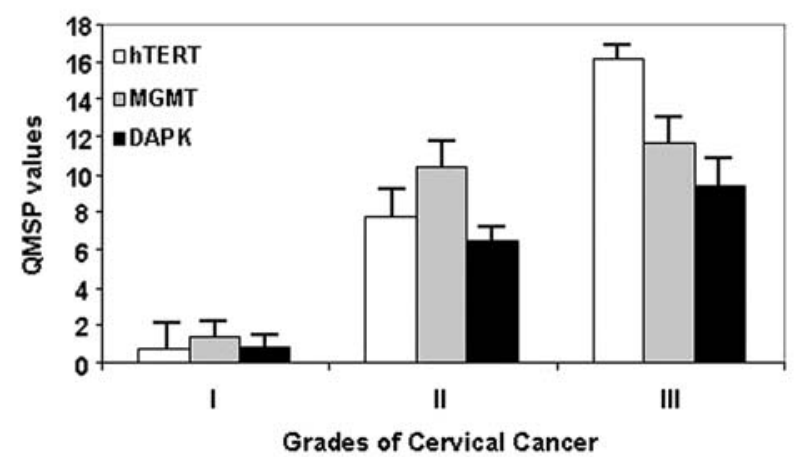

Figure 2. Correlation of hTERT, MGMT and DAPK promoter methylation status with stage and grade in cervical carcinomas. (a) DAPK and MGMT promoters hypermethylation is able to distinguish significantly $(\mathrm{p}<0.001)$ the stages of the disease, whereas hTERT could not distinguish stage II from stage III-IV cervical carcinomas $(\mathrm{p}>0.05)$. (b) Correlation of hTERT, MGMT and DAPK methylation status with grade in cervical cancer. All three genes can distinguish between different grades in cervical cancer, with hTERT showing a better association $(\mathrm{p}<0.0001)$.

methylation status showed $86.8 \%$ sensitivity in the detection of cancer and $100 \%$ in HGSIL (CIN3) detection.

Promoter hypermethylation in relation to histological findings. To determine a possible association between methylation status and histological outcome (CIN1, CIN2 and CIN3), the mean QMSP values of methylation in each gene was evaluated. Mean normalized QMSP values of hTERT promoter methylation were 2.05 (range 0-6.7), 3.2 (range 0-11.2) and 6.02 (range 0-13.2) in CIN 1, CIN2, CIN3, respectively. A significant difference was observed between CIN1 and cancer, CIN1 and CIN3, CIN2 and CIN3, CIN2 and cancer and between CIN3 and cancer $(\mathrm{p}<0.05)$.

Mean QMSP values of MGMT promoter methylation were 0.56 (range 0-3.1), 0.65 (range 0-4.3) and 4.3 (range 0-11.2) in CIN1, CIN2, CIN3, respectively. A significant difference was also observed between CIN1 and cancer, CIN2 and CIN3, CIN2 and cancer, CIN3 and cancer as well as between CIN2 and CIN3 and CIN1 and CIN3 (p<0.05).

Moreover, DAPK mean QSP values were 0.85 (range 0-3.1), 3.6 (range 0-7.7) and 5.8 (range 0-21.2) in CIN1, CIN2, CIN3, respectively. A significant difference was observed between CIN1 and cancer, CIN1 and CIN3, as well as between normal and CIN3, normal and CIN2 and CIN1 and CIN2. 
Promoter hypermethylation in relation to HPV infection. To determine whether there is an association between methylation status of each gene and HPV detection, the mean QMSP values of methylation in each gene were compared to HPV presence or type. Oncogenic HPV types (type 16 and 18) were detected in $1 / 5(20 \%)$ with ASCUS, $4 / 11(36.4 \%)$ with LGSIL, 8/11 (72.7\%) with HGSIL and in 26/32 (81.25\%) cancers cases. There were no significant associations between the presence or types of HPV and methylation status for all genes $(\mathrm{p}>0.05)$.

Correlation of DNA methylation levels and clinicopathological parameters in cervical cancer samples. In cervical samples, the detection of aberrant methylation in each of the three genes studied was correlated with tumor stage and grade of the disease. Specifically, DAPK and MGMT promoter hypermethylation seemed to be able to distinguish significantly the stages of the disease, whereas hTERT could not ( $>>0.05$ ) (Fig. 2a). Higher stage (III-IV) tumors exhibited an increased overall frequency of promoter methylation (Table III). On the other hand, all three genes seemed to distinguish between different grades in cervical cancer, with hTERT showing a better association $(\mathrm{p}<0.0001)$ (Fig. 2b).

\section{Discussion}

Research in the last few years clearly proved that epigenetic events and particularly methylation are involved in cancer initiation and progression and that their expression profiling can be exploited for the classification, diagnosis and prediction of human malignancies (25-31).

In the present study, we investigated the methylation profiles in the promoter region of hTERT, MGMT and DAPK genes in normal, premalignant and cervical cancer specimens, as the existing data on the methylation status of these genes is limited and results are conflicting (22,32-36). hTERT gene plays a critical role in cell division and serves as a mitotic clock for cell proliferation (17). In our study we found that the mean levels of hTERT promoter methylation were increased with increasing cytological and histological severity in agreement with previous relative reports and also that QMSP hTERT methylation values could distinguish normal from premalignant and cancer tissues, as well as premalignant from cancer cervical tissues $(25,37)$.

The MGMT gene product removes mutagenic and cytotoxic adducts from $\mathrm{O}^{6}$-guanine in DNA, the preferred point of attack of many carcinogens and alkylating chemotherapeutic agents (38). As a consequence, its lack of expression produces opposite effects for cancer development and progression. We observed that MGMT gene showed higher levels of hypermethylation in premalignant and cancer compared to normal tissues, distinguishing thus premalignant from cancer tissues during disease progression. This finding is in agreement with previous relative studies, where it has been suggested that higher levels of MGMT hypermethylation are related to increased susceptibility in cancer development $(21,22,24-26,32)$.

DAP-kinase (DAPK) is a positive mediator of programmed cell death induced by $\gamma$-interferon (39). Loss of DAPK expression has been shown to occur in a number of malig- nancies including cervical cancer by enhancing the metastatic potential of cancer cells (40-44). In previous studies DAPK promoter hypermethylation was observed in $2 \%$ of normal, $17-55 \%$ of HGSILs samples and $45-100 \%$ of cancer cases (21,34-36,39-42,45-48). In the present study DAPK promoter hypermethylation was detected in $66.7 \%$ of premalignant lesions, percentage which is higher than in previous reports (34-36) and it was able to distinguish early from late stages of cervical oncogenesis progression. DAPK promoter methylation status seemed to be the best cervical oncogenesis quantitative and qualitative marker, as it was the only marker that was negative in normal tissues. The higher hypermethylation values of hTERT, MGMT and DAPK, observed in the present study, could be attributed to the use of the sensitive Quantitative Methylation Specific (QMSP) method in contrast to conventional MSP methods used in previous studies $(22,27)$.

Moreover, we evaluated for the first time to our knowledge, the combination of hTERT, DAPK and MGMT hypermethylation profiles in cervical carcinogenic progression. We observed that the hypermethylation profile of these 3 genes combined showed a higher sensitivity in the detection of cancer $(86.8 \%)$ and HGSIL (CIN3) (100\%), compared to the combination of two of them or each gene separately. As promoter hypermethylation levels tended to increase from normal to dysplastic and cancer specimens, it is suggested that hypermethylation of these three genes could be involved in cervical progression.

Furthermore, we also observed for the first time, an association between hypermethylation of hTERT, DAPK and MGMT genes and disease grade in cancer samples, with hTERT showing the best correlation and the highest sensitivity $(80 \%)$, suggesting the possible involvement of hTERT methylation levels as a potential molecular marker in gynecological cancer.

In conclusion, we showed that promoter hypermethylation of hTERT, DAPK and MGMT genes increased with disease progression suggesting that hypermethylation could be a mechanism involved during the development of cervical carcinogenesis. Although identifying abnormal methylation events is promising, further efforts are needed to identify and validate the optimal combination of genes that could be used as adjunctive molecular markers to current screening approaches.

\section{Acknowledgements}

The authors would like to thank Ms. Chrysa Cratsagoni for sample collection.

\section{References}

1. Jemal A, Thomas A, Murray, et al: Cancer Statistics, 2002 CA. Cancer J Clin 52: 23-47, 2002.

2. Vizcaino AP, Moreno V, Bosch FX, Muñoz N, Barros-Dios XM, Borras $\mathrm{J}$ and Parkin DM: International trends in incidence of cervical cancer: II. Squamous-cell carcinoma. Int J Cancer 86: 429-435, 2000.

3. Wright TC, Ferenczy A and Kurman R: Carcinoma and other tumors of the cervix. In: Pathology of the Female Genital Tract. Kurman RJ (ed). Springer-Verlag, New York, pp279-326, 1994.

4. Zur Hausen H and Rosl F: Pathogenesis of cancer of the cervix. Cold Spring Harb Symp Quant Biol 9: 623-628, 1994. 
5. Bosch FX, Manos MM, Muñoz N, et al: Prevalence of human papillomavirus in cervical cancer: a worldwide perspective. International Biological Study on Cervical Cancer (IBSCC) Study Group. J Natl Cancer Inst 87: 796-802, 1995.

6. Schiffman MH and Castle P: Epidemiologic studies of a necessary causal risk factor: human papillomavirus infection and cervical neoplasia. J Natl Cancer Inst 95: E2, 1994

7. Waggoner SE: Cervical cancer. Lancet 361: 2217-2225, 2003.

8. Koss LG: Cervical (Pap) smear. New directions. Cancer 71: 1406-1412, 1993.

9. Kulasingam SL, Hughes JP, Kiviat NB, Mao C, Weiss NS, Kuypers JM and Koutsky LA: Evaluation of human papillomavirus testing in primary screening for cervical abnormalities comparison of sensitivity, specificity and frequency of referral. JAMA 288: 1749-1757, 2002.

10. Jones PA and Baylin SB: The fundamental role of epigenetic events in cancer. Nat Rev Genet 3: 415-428, 2002.

11. Baldwin RL, Nemeth E, Tran HShvartsman H, Cass I, Narod S and Karlan BY: BRCA1 promoter region hypermethylation in ovarian carcinoma: a population-based study. Cancer Res 60: 5329-5333, 2004

12. Esteller M, Silva JM, Dominguez G, et al: Promoter hypermethylation and BRCA1 inactivation in sporadic breast and ovarian tumors. J Natl Cancer Inst 92: 564-569, 2000.

13. Salvesen HB, Macdonald N, Ryan A, Jacobs IJ, Lynch ED, Akslen LA and Das S: PTEN methylation is associated with advanced stage and microsatellite instability in endometrial carcinoma. Int J Cancer 91: 22-26, 2001.

14. Zysman M, Saka A, Millar A, Knight J, Chapman W and Bapat B: Methylation of adenomatous polyposis coli in endometrial cancer occurs more frequently in tumors with microsatellite instability phenotype. Cancer Res 62: 3663-3666, 2002.

15. Yang HJ, Liu VWS, Wang Y, et al: Detection of hypermethylated genes in tumour and plasma of cervical cancer patients. Gynecol Oncol 93: 435-440, 2004.

16. Dueñas-González A, Lizano M, Candelaria M, Cetina L, Arce $\mathrm{C}$ and Cervera E: Epigenetics of cervical cancer. An overview and therapeutic perspectives. Mol Cancer 4: 38, 2005.

17. Poole CJ, Andrews GL and Tollefsbol TO: Activity, function, and gene regulation of the catalytic subunit of telomerase (hTERT). Gene 269: 1-12, 2001.

18. Cohen SB, Graham ME, Lovrecz GO, Bache N, Robinson PJ and Reddel RR: Protein composition of catalytically active human telomerase from immortal cells. Science 315: 1850-1853, 2007.

19. Shohat G, Shani G, Eisenstein M and Kimchi A: The DAPkinase family of proteins: study of a novel group of calciumregulated death-promoting kinases. Biochem Biophys Acta 600: 45-50, 2002.

20. Hegi ME, Diserens AC, Gorlia T, et al: MGMT gene silencing and benefit from temozolomide in glioblastoma. N Engl J Med 352: 997-1003, 2005.

21. Berek J and Hacker N: In: Practical Gynecology Oncology. 3rd edition, Ch. 9. Berek J and Hacker N (eds). Lippincott Williams \& Wilkins, Philadelphia, pp345-406, 2000.

22. Benedet JL: Progress in gynecologic cancer detection and treatment. Int J Gynaecol Obstet 70: 135-147, 2000.

23. Tsezou A, Oikonomou P, Kollia P, Mademtzis I, Kostopoulou E, Messinis I and Vamvakopoulos N: The role of human telomerase catalytic subunit mRNA expression in cervical dysplasias. Exp Biol Med (Maywood) 230: 263-270, 2005.

24. Iliopoulos D, Guler G, Han SY, et al: Fragile genes as biomarkers: epigenetic control of WWOX and FHIT in lung, breast and bladder cancer. Oncogene 24: 1625-1633, 2005.

25. Widschwendter A, Muller HM, Fiegl H, et al: DNA methylation in serum and tumors of cervical cancer patients. Clin Cancer Res 10: 565-571, 2004.

26. Hiraga J, Kinoshita T, Ohno T, et al: Promoter hypermethylation of the DNA-repair gene O6-methylguanine-DNA methyltransferase and p53 mutation in diffuse large B-cell lymphoma. Int J Hematol 84: 248-255, 2006.

27. Yang HJ, Liu VWS, Wang Y, Tsang PC and Ngan HY: Differential DNA methylation profiles in gynecological cancers and correlation with clinicopathological data. Cancer 6: 212-222, 2006

28. Catto JWF, Azzouzi AR, Rehman I, et al: Promoter hypermethylation is associated with tumor location, stage, and subsequent progression in transitional cell carcinoma. J Clin Oncol 23: 2903-2910, 2005.
29. Hoque MO, Feng Q, Toure P, et al: Detection of aberrant methylation of four genes in plasma DNA for the detection of breast cancer. J Clin Oncol 24: 4263-4269, 2006.

30. Doorn van R, Zoutman WH, Dijkman R, et al: Epigenetic profling of cutaneous T-cell lymphoma: promoter hypermethylation of multiple tumor suppressor genes including BCL7a, PTPRG, and p73. J Clin Oncol 23: 3886-3898, 2005.

31. Kang S, Kim WJ, Kang GH, et al: Comparison of DNA hypermethylation patterns in different types of uterine cancer: cervical squamous cell carcinoma, cervical adenocarcinoma and endometrial adenocarcinoma. Int J Cancer 118: 2168-2171, 2006.

32. Virmani AK, Muller C and Rathi A: Aberrant methylation during cervical carcinogenesis. Clin Cancer Res 7: 584-589, 2001.

33. Oikonomou P, Messinis I and Tsezou A: DNA methylation is not likely to be responsible for hTERT expression in premalignant cervical lesions. Exp Biol Med (Maywood) 232: 881-886, 2007.

34. Wisman GB, Nijhuis ER, Hoque MO, et al: Assessment of gene promoter hypermethylation for detection of cervical neoplasia. Int J Cancer 15 119: 1908-1914, 2006.

35. Kahn SL, Ronnett BM, Gravitt PE, et al: Quantitative methylation- specific PCR for the detection of aberrant DNA methylation in liquid-based Pap tests. Cancer 114: 57-64, 2008.

36. Shivapurkar N, Sherman ME, Stastny V, et al: Evaluation of candidate methylation markers to detect cervical neoplasia. Gynecol Oncol 107: 549-553, 2007.

37. Widschwendter A, Muller HM, Hubalek MM, et al: Methylation status and expression human telomerase reverse transcriptase in ovarian and cervical cancer. Gynec Oncol 93: 407-416, 2004.

38. Esteller M and Herman JG: Generating mutations but providing chemosensitivity: the role of O6-methylguanine DNA methyltransferase in human cancer. Oncogene 23: 1-8, 2004.

39. Deiss LP, Feinstein E, Berissi H, Cohen O and Kimchi A: Identification of a novel serine/threonine kinase and a novel $15-\mathrm{kD}$ protein as potential mediators of the gamma interferoninduced cell death. Genes Dev 9: 15-30, 1995.

40. Feng Q, Balasubramanian A, Hawes SE, et al: Detection of hypermethylated genes in women with and without cervical neoplasia. Natl Cancer Inst 97: 273-282, 2005.

41. Jeong DH, Youm MY, Kim YN, Lee KB, Sung MS, Yoon HK and Kim KT: Promoter methylation of p16, DAPK, CDH1, and TIMP-3 genes in cervical cancer: correlation with clinicopathologic characteristics. Int J Gynecol Cancer 16: 1234-1240, 2006.

42. Reesink-Peters N, Wisman GBA, Jéronimo C, et al: Detecting cervical cancer by quantitative promoter hypermethylation assay on cervical scrapings: a feasibility study. Mol Cancer Res 2: 289-295, 2004.

43. Narayan G, Arias-Pulido H, Koul S, et al: Frequent promoter methylation of CDH1, DAPK, RARB, and HIC1 genes in carcinoma of cervix uteri: its relationship to clinical outcome. Mol Cancer 2: 24, 2003.

44. Simpson DJ, Clayton RN and Farrell WE: Preferential loss of death associated protein kinase expression in invasive pituitary tumours is associated with either $\mathrm{CpG}$ island methylation or homozygous deletion. Oncogene 21: 1217-1222, 2002.

45. Kang S, Kim JW, Kang GH, Park NH, Song YS, Kang SB and Lee HP: Polymorphism in folate- and methionine-metabolizing enzyme and aberrant $\mathrm{CpG}$ island hypermethylation in uterine cervical cancer. Gynecol Oncol 96: 173-180, 2005.

46. Terra AP, Murta EF, Maluf PJ, Caballero OL, Brait M and Adad SJ: Aberrant promoter methylation can be useful as a marker of recurrent disease in patients with cervical intraepithelial neoplasia grade III. Tumori 93: 572-579, 2007.

47. Jarboe EA, Liaw KL, Thompson LC, et al: Analysis of telomerase as a diagnostic biomarker of cervical dysplasia and carcinoma. Oncogene 21: 664-673, 2002.

48. Snijders PJF, Van Duin M, Walboomers MMJ, et al: Telomerase activity exclusively in cervical carcinomas and a subset of cervical intraepithelial neoplasia grade III lesions: strong association with elevated messenger RNA levels of its catalytic subunit and high-risk human papillomavirus DNA. Cancer Res 58: 3812-3818, 1998. 